\title{
An explicit time evolution method for acoustic wave propagation
}

\author{
Huafeng Liu', Nanxun Dai ${ }^{2}$, Fenglin $\mathrm{Niu}^{3}$, and Wei $\mathrm{Wu}^{2}$
}

\begin{abstract}
Cost-effective waveform modeling is the key to practical reverse time migration (RTM) and full-waveform inversion (FWI) implementations. We evaluated an explicit time evolution (ETE) method to efficiently simulate wave propagation in acoustic media with high temporal accuracy. We started from the constant-density acoustic wave equation and obtained an analytical time-marching scheme in the wavenumber domain. We then formulated an ETE scheme in the time-space domain by introducing a cosine function approximation. Although the ETE operator appears to be similar to the second-order temporal finite-difference (FD) operator, the exact nature of the ETE formula ensures high accuracy in time. We further introduced a set of optimum stencils and coefficients by minimizing evolution errors in a least-squares sense. Our numerical tests indicated that ETE can achieve similar waveform accuracy as FD with four times larger time steps. Meanwhile, the compact ETE operator keeps the computation efficient. The efficiency and capability to handle complex velocity field make ETE an attractive engine in acoustic RTM and FWI.
\end{abstract}

\section{INTRODUCTION}

Increased computer performance has enabled applications of reverse-time migration (RTM) (Baysal et al., 1983; McMechan, 1983; Whitmore, 1983) and full-waveform inversion (FWI) (Tarantola, 1984) in seismic exploration. Nevertheless, the computational cost of RTM and FWI is still a bottleneck to wider application. The most computationally expensive part that RTM and FWI share is a forward modeling engine that numerically simulates wavefields. Hence, a full-waveform modeling method with high accuracy and efficiency is key to improving the affordability of RTM and FWI.

To model full-wave propagation in heterogeneous media, numerical methods such as finite-difference (FD), pseudospectral, and finite-element are often used (Carcione et al., 2002). The FD approach is perhaps the most common forward modeling method employed in current implementations of RTM and FWI. The FD method approximates the spatial and temporal derivatives of the wave equation using Taylor series expansions, the order of which controls the accuracy. A second-order temporal scheme is often favored in FD because direct implementation of higher order temporal terms requires significantly more memory. The second-order temporal approximation is reasonably accurate only if the temporal sampling interval is fine. Hence, the second-order temporal FD method requires time steps that are significantly finer than the Von Neumann stability requirement to remain reasonably accurate through time. Etgen (1986) and Dablain (1986) use the Lax-Wendroff method to approximate the fourth-order temporal derivative by spatial terms and successfully substitute the computation time with a memory access cost. Spectral methods (Tal-Ezer et al., 1987; Etgen, 1989; Pestana and Stoffa, 2010; Tessmer, 2011) can achieve high temporal accuracy by approximating the time evolution operator with Chebyshev polynomials. The corresponding high temporal accuracy allows coarser time steps than the FD method. However, the required spatial Fourier transforms make the cost per time step significantly more than in the FD method. In acoustic media, the time evolution of the wavefield can be formulated analytically by an integral of the product of the current wavefield and a cosine function in wavenumber domain, known as the Fourier integral (e.g., Soubaras and Zhang, 2008; Song and Fomel, 2011; Alkhalifah, 2013). Soubaras and Zhang (2008) first propose to approximate the cosine function in the Fourier integral by a polynomial, resulting in a two-step explicit marching scheme that allows large time steps. Zhang and Zhang (2009) further introduce a complex wavefield and suggest a one-step extrapolation by using multiple times of fast Fourier transforms (FFTs). Song and Fomel (2011)

Manuscript received by the Editor 24 February 2013; revised manuscript received 27 October 2013; published online 12 March 2014.

${ }^{1}$ Rice University, Department of Earth Science, Houston, Texas, USA. E-mail: lhffelix@ gmail.com.

${ }^{2}$ BGP International, Inc., Houston, Texas, USA. E-mail: dainanxun@gmail.com; weiwu1982@yahoo.com.cn.

${ }^{3}$ China University of Petroleum, State Key Laboratory of Petroleum Resource and Prospecting, and Unconventional Natural Gas Institute, Beijing, China and Rice University, Department of Earth Science, Houston, Texas, USA. E-mail: niu@cup.edu.cn.

(C) 2014 Society of Exploration Geophysicists. All rights reserved. 
develop a Fourier FD method to better handle variable velocity with one FFT pair in each time step. Fomel et al. (2013) propose a more inclusive seismic lowrank wave extrapolation method that uses a small set of representative spatial locations and wavenumbers to approximate the integral with a small number of FFTs. Song et al. (2013) further derive an explicit FD method from the lowrank approximation. Instead of focusing on reducing the number of FFTs, Alkhalifah (2013) introduces a residual extrapolation operator in the wavenumber domain that allows us to accurately extrapolate waves with large time steps. Compared to the FD method, FFTbased methods can achieve higher accuracy by requiring more computations in FFTs. One drawback, however, is that the FFT-based methods require additional treatments to suppress the wrap-around effect caused by the periodic source assumption.

Our target is to achieve high temporal accuracy efficiently without FFT. Starting from the constant-density acoustic wave equation, we formulate an explicit time evolution (ETE) scheme by introducing a cosine function approximation to the exact time evolution solution. Benefiting from an explicit FD-like scheme, the ETE method does not have a wrap-around effect and is adaptive to boundary conditions similar to the FD method. Furthermore, the cosine function approximation can be achieved fairly accurately, ensuring the accuracy of the ETE operator. By optimizing the stencil and coefficients in the ETE scheme, we are able to achieve high temporal accuracy efficiently. Finally, we use synthetic examples to show that the ETE method can achieve high accuracy in waveform modeling and is feasible in RTM implementation.

\section{METHOD}

\section{Theory}

We start from the constant-density acoustic-wave equation in the time-space domain:

$$
\frac{\partial^{2} p(\mathbf{x}, t)}{\partial t^{2}}=v(\mathbf{x})^{2} \nabla^{2} p(\mathbf{x}, t),
$$

where $\mathbf{x}=(x, y, z), \nabla^{2}$ is the spatial Laplacian operator, $p(\mathbf{x}, t)$ is the pressure field, and $v(\mathbf{x})$ is the seismic velocity. Assuming a constant velocity $v$, the acoustic-wave equation 1 can be written in the wavenumber domain as

$$
\frac{d^{2} \tilde{p}(\mathbf{k}, t)}{d t^{2}}=v^{2}|k|^{2} \tilde{p}(\mathbf{k}, t),
$$

with a spatial Fourier transform:

$$
\tilde{p}(\mathbf{k}, t)=\int_{-\infty}^{+\infty} p(\mathbf{x}, t) e^{-i \mathbf{k} \cdot \mathbf{x}} \mathrm{d} \mathbf{x}
$$

where $\mathbf{k}=\left(k_{x}, k_{y}, k_{z}\right)$ is the wavenumber vector. Equation 2 has analytical solutions at arbitrary times:

$$
\tilde{p}(\mathbf{k}, t)=A e^{i|\mathbf{k}| v t}+B e^{-i|\mathbf{k}| v t} .
$$

$A$ and $B$ are arbitrary coefficients. Equation 4 holds for $t+\Delta t$ and $t-\Delta t$ :

$$
\tilde{p}(\mathbf{k}, t \pm \Delta t)=A e^{i|\mathbf{k}| v(t \pm \Delta t)}+B e^{-i|\mathbf{k}| v(t \pm \Delta t)} .
$$

Combining the wavefield at $t-\Delta t$ and $t+\Delta t$, we find the time evolution is irrelevant to $A$ or $B$ :

$$
\tilde{p}(\mathbf{k}, t+\Delta t)+\tilde{p}(\mathbf{k}, t-\Delta t)=2 \cos (|\mathbf{k}| v \Delta t) \tilde{p}(\mathbf{k}, t) .
$$

Equation 6 describes an accurate time-marching scheme in the wavenumber domain (Etgen, 1989; Soubaras and Zhang, 2008). If we approximate the cosine function by Taylor's series expansion at $|\mathbf{k}| v \Delta t=0$ and truncate it to the second order

$$
\cos (|\mathbf{k}| v \Delta t)=1-\frac{|\mathbf{k}|^{2} v^{2} \Delta t^{2}}{2}+O\left((|\mathbf{k}| v \Delta t)^{4}\right)
$$

a familiar pseudospectral method with second-order temporal accuracy can be derived:

$$
\frac{\tilde{p}(|\mathbf{k}|, t+\Delta t)+\tilde{p}(|\mathbf{k}|, t-\Delta t)-2 \tilde{p}(|\mathbf{k}|, t)}{\Delta t^{2}} \approx-v^{2}|\mathbf{k}|^{2} \tilde{p}(|\mathbf{k}|, t) .
$$

Equation 8 is a spatially accurate scheme with low temporal accuracy. To balance the spatial and temporal accuracy, an explicit FD scheme with arbitrary spatial accuracy can be derived by introducing FD approximating to the spatial operator in Equation 8.

Fourier methods (Soubaras and Zhang, 2008; Zhang and Zhang, 2009; Pestana and Stoffa, 2010; Song and Fomel, 2011; Tessmer, 2011; Alkhalifah, 2013; Fomel et al., 2013) have been developed to solve equation 6 effectively. To explore the possibility of achieving high temporal accuracy without FFTs, we derive an ETE scheme from equation 6 using values at a set of grid points (stencil):

$$
\begin{aligned}
p(\mathbf{x}, t+\Delta t)= & -p(\mathbf{x}, t-\Delta t) \\
& +\sum_{m=0}^{N_{s}-1} C\left(\mathbf{x}, \Delta \mathbf{x}_{m}\right) p\left(\mathbf{x}+\Delta \mathbf{x}_{m}, t\right)+E(\mathbf{x}, t),
\end{aligned}
$$

where $m$ is the grid index, $\Delta \mathbf{x}_{m}=\mathbf{x}_{m}-\mathbf{x}$ is the location difference between grid $m$ and the target location $\mathbf{x}, C\left(\mathbf{x}, \Delta \mathbf{x}_{m}\right)$ is the weighting coefficient at each stencil point, $N_{s}$ is the total number of points in the stencil, and $E(\mathbf{x}, t)$ is the error in time evolution. If we choose an origin-symmetric stencil around $\mathbf{x}$ and use the property of the Fourier transform, equation 9 becomes

$$
\begin{aligned}
& p(\mathbf{x}, t+\Delta t) \\
& =-p(\mathbf{x}, t-\Delta t)+\sum_{m=0}^{M-1} C\left(\mathbf{x}, \Delta \mathbf{x}_{m}\right) \int\left(e^{i \mathbf{k} \cdot\left(\mathbf{x}+\Delta \mathbf{x}_{m}\right)}\right. \\
& \left.+e^{i \mathbf{k} \cdot\left(\mathbf{x}-\Delta \mathbf{x}_{m}\right)}\right) \tilde{p}(\mathbf{k}, t) \mathrm{d} \mathbf{k}+E(\mathbf{x}, t) \\
& =-p(\mathbf{x}, t-\Delta t) \\
& +\sum_{m=0}^{M-1} C\left(\mathbf{x}, \Delta \mathbf{x}_{m}\right) \int 2 \cos \left(\mathbf{k} \cdot \Delta \mathbf{x}_{m}\right) \tilde{p}(\mathbf{k}, t) e^{i \mathbf{k} \cdot \mathbf{x}} \mathrm{d} \mathbf{k}+E(\mathbf{x}, t) \\
& =-p(\mathbf{x}, t-\Delta t) \\
& +\int\left(\sum_{m=0}^{M-1} 2 C\left(\mathbf{x}, \Delta \mathbf{x}_{m}\right) \cos \left(\mathbf{k} \cdot \Delta \mathbf{x}_{m}\right)\right) \tilde{p}(\mathbf{k}, t) e^{i \mathbf{k} \cdot \mathbf{x}} \mathrm{d} \mathbf{k}+E(\mathbf{x}, t) .
\end{aligned}
$$


Here, $M=\left(N_{s}+1\right) / 2$ is the number of independent grid points in an origin-symmetric stencil. We further represent the error term as

$$
E(\mathbf{x}, t)=\int 2 \tilde{p}(\mathbf{k}, t) E(\mathbf{x}, \mathbf{k}) e^{i \mathbf{k} \cdot \mathbf{x}} \mathrm{d} \mathbf{k},
$$

where $E(\mathbf{x}, \mathbf{k})$ is the wavenumber domain misfit at $\mathbf{x}$. Through equations 10 and 11, we find that the ETE scheme (equation 9) will be associated with the accurate time marching scheme (equation 6) in the space domain, if we introduce a cosine function approximation:

$$
\cos (|\mathbf{k}| v \Delta t)=\sum_{m=0}^{M-1} C\left(\mathbf{x}, \Delta \mathbf{x}_{m}\right) \cos \left(\mathbf{k} \cdot \Delta \mathbf{x}_{m}\right)+E(\mathbf{x}, \mathbf{k}) .
$$

Given the Von Neumann stability condition for explicit schemes:

$$
\Delta t<\frac{\min \left(\Delta \mathbf{x}_{m}\right)}{\sqrt{n} \times \max (v(\mathbf{x}))},
$$

where $n$ is the spatial dimension of the medium, the wavefield advance is limited to less than one grid point within each time step. Under this limitation, only a localized constant velocity is required. Hence, we can extend equation 12 in a complex velocity media as

$$
\cos (|\mathbf{k}| v(\mathbf{x}) \Delta t)=\sum_{m=0}^{M-1} C\left(\mathbf{x}, \Delta \mathbf{x}_{m}\right) \cos \left(\mathbf{k} \cdot \Delta \mathbf{x}_{m}\right)+E(\mathbf{x}, \mathbf{k})
$$

We have reformulated the accurate time marching scheme (equation 6) into an ETE scheme (equation 9), in which the cosine function is approximated by a weighted summation of cosine functions evaluated at stencil grids, as shown in equation 14 . The evolution error in each time step $E(\mathbf{x}, t)$ is explicitly related to the error in fitting the cosine function at all wavenumbers $E(\mathbf{x}, \mathbf{k})$. Thus, the accuracy of the time evolution is solely determined by the fit of the cosine function at each wavenumber. For a given velocity field and spatial discretization, the cosine function fit is controlled by the selection of the grid distribution (stencil shape) $\Delta \mathbf{x}_{m}$ and the corresponding coefficients $C\left(\mathbf{x}, \Delta \mathbf{x}_{m}\right)$ at each spatial location.

\section{Optimum stencil and coefficients}

For any given stencil, we seek coefficients that minimize the error in the wavenumber domain $E(\mathbf{x}, \mathbf{k})$ at each spatial location $\mathbf{x}$. This is a typical optimization problem that can be formulated by minimizing the L2-norm of the error:

$$
\begin{aligned}
& \min \left(\|E(\mathbf{k})\|_{2}\right) \\
& =\min \left(\left\|\cos (|\mathbf{k}| v(\mathbf{x}) \Delta t)-\sum_{m=1}^{M} C\left(\mathbf{x}, \Delta \mathbf{x}_{m}\right) \cos \left(\mathbf{k} \cdot \Delta \mathbf{x}_{m}\right)\right\|_{2}\right) .
\end{aligned}
$$

More specifically, the coefficients at each spatial location $\mathbf{x}$ can be independently obtained by a vector-matrix inverse problem:

$$
\begin{gathered}
{\left[\begin{array}{cccc}
\cos \left(\mathbf{k}_{1} \cdot \Delta \mathbf{x}_{1}\right) & \cos \left(\mathbf{k}_{1} \cdot \Delta \mathbf{x}_{2}\right) & \ldots & \cos \left(\mathbf{k}_{1} \cdot \Delta \mathbf{x}_{M}\right) \\
\cos \left(\mathbf{k}_{2} \cdot \Delta \mathbf{x}_{1}\right) & \cos \left(\mathbf{k}_{2} \cdot \Delta \mathbf{x}_{2}\right) & \ldots & \cos \left(\mathbf{k}_{2} \cdot \Delta \mathbf{x}_{M}\right) \\
\ldots & \ldots & \ldots & \ldots \\
\cos \left(\mathbf{k}_{N_{k}} \cdot \Delta \mathbf{x}_{1}\right) & \cos \left(\mathbf{k}_{N_{k}} \cdot \Delta \mathbf{x}_{2}\right) & \ldots & \cos \left(\mathbf{k}_{N_{k}} \cdot \Delta \mathbf{x}_{M}\right)
\end{array}\right]} \\
\times\left[\begin{array}{c}
C\left(\mathbf{x}, \Delta \mathbf{x}_{1}\right) \\
C\left(\mathbf{x}, \Delta \mathbf{x}_{2}\right) \\
\ldots \\
C\left(\mathbf{x}, \Delta \mathbf{x}_{M}\right)
\end{array}\right]=\left[\begin{array}{c}
\cos \left(\left|\mathbf{k}_{1}\right| v(\mathbf{x}) \Delta t\right) \\
\cos \left(\left|\mathbf{k}_{2}\right| v(\mathbf{x}) \Delta t\right) \\
\ldots \\
\cos \left(\left|\mathbf{k}_{N_{k}}\right| v(\mathbf{x}) \Delta t\right)
\end{array}\right],
\end{gathered}
$$

where $N_{k}$ is the number of wavenumbers. At each spatial location, we solve for $C\left(\mathbf{x}, \Delta \mathbf{x}_{m}\right)$ by QR factorization.

Naturally, we seek a stencil that balances accuracy and efficiency. For a grid spacing $h$ and $\gamma=(v \Delta t) / h$ the cosine-fitting equation 14 can be characterized by a scalar equation:

$$
\cos (\gamma h|\mathbf{k}|)=c_{0}+\sum_{i=1}^{N_{a}} c_{i} \cos \left(n_{i} h|\mathbf{k}|\right)+\sum_{j=1}^{N_{o}} c_{j} \cos \left(r_{j} h|\mathbf{k}|\right),
$$

where $n_{i}$ and $r_{i}$ are integers and real numbers $(>1)$ associated with the axial and off-axial stencil points, respectively; $c_{i}$ and $c_{j}$ are the corresponding coefficients ( $c_{o}$ is the coefficient of the central point); and $N_{a}$ and $N_{o}$ are the half total numbers of the axial (except for the central point) and off-axial points, respectively.

The conventional FD method is a special case of time evolution, which has only axial stencil points $\left(N_{o}=0\right)$ and uses FD approximation to solve the coefficients. The $L$ th-order FD has $L$ stencil points in each spatial dimension beside the central point, which makes $N_{s}=n L+l$ (Figure 1a), where $n$ is the medium dimension. Dai et al. (2012) compute the least-squares coefficients of the FD stencil, which we refer as the "optimized FD" (OFD) method. We first compare the OFD method with the conventional FD method. We set $N_{a}=8$ and $N_{o}=0$ and compare the misfits in fitting the cosine function (Figure $2 \mathrm{a}$ and $2 \mathrm{~b}$ ) using the same base functions but different coefficients corresponding to the OFD and FD methods. Overall, the OFD method fits the cosine function better than the FD method does. However, for small wavenumbers, the OFD exhibits large oscillations that are not desirable. As mentioned above, the coefficient $\left(r_{j}\right)$ of off-axial stencil points is usually not an integer. For instance, if we set $N_{a}=8, N_{o}=2, r_{1}=r_{2}=\sqrt{2}$ and solve for the corresponding least-squares coefficients and then compare the corresponding cosine function curve and misfits (ETE in Figure $2 \mathrm{a}$ and $2 \mathrm{~b}$, respectively) for FD and OFD, we see that the additional base function significantly reduces the misfit of the cosine function. To optimize the choice of the additional base functions, we analyze the dependence of misfit on the distance number $r$. Figure $2 \mathrm{c}$ shows that the misfit at all wavenumbers increases with increasing $r$. Because the distance number, $r$, of the off-axial points is always greater than 1 and the Von Neumann stability condition in equation 13 ensures $\gamma<\frac{1}{\sqrt{n}} \leq 1, r \neq \gamma$. A stencil that includes offaxial points being closest to its center thus appears to be the optimum selection (Figure 1b). 
To summarize, in fitting the cosine function in equation 17 , we have shown that adding a noninteger $r$ can significantly reduce the misfit and that a smaller $r$ is more effective in reducing fitting errors. In 2D and 3D problems, including off-axial stencil points with minimal distances to the center (Figure 1b) can effectively reduce the fitting error in equation 14 . We refer the method with optimized stencil and corresponding least-squares coefficients as the ETE method.

\section{ETE implementation}

The most straightforward way to obtain ETE coefficients is solving the inverse problem (equation 16) at all spatial locations with a computational complexity of $O\left(N_{s}^{2} \cdot N_{k} \times N_{x}\right)$. In our ETE implementation, we reduce the cost of computing the ETE coefficients by using representative wavenumbers and velocities.

First, we degenerate the inverse problem (equation 15) by using representative wavenumbers. In equation 16 , the target and base functions are smooth cosine domes and cosine cylinders, respectively. As a result, it is not efficient to fit the cosine function at all wavenumbers. If we resample the wavenumber using $N_{k_{i}}$ representative wavenumbers in each dimension, the total wavenumbers are reduced to $N_{k_{i}}^{n}$. As a result, the computational cost is reduced to $O\left(N_{s}^{2} \cdot N_{k_{i}}^{n} \times N_{x}\right)$, which is independent of spatial discretization. In an $L$ th-order ETE scheme, the definition range of the target and base cosine functions is bounded by

$$
|\mathbf{k}| v \Delta t<|\mathbf{k}|\left|\Delta \mathbf{x}_{m}\right|<=\left|\max (\mathbf{k}) \times\left(\frac{L}{2} h\right)\right|<\frac{L}{4} .
$$

Therefore, the maximum resampling interval is upper bounded by $\frac{L+2}{2 N_{k_{i}}}$ in each dimension. For instance, if we set the sampling interval of the dimensionless variable $|\mathbf{k}|\left|\Delta \mathbf{x}_{m}\right|$ in the cosine function as 0.1 in a $2 \mathrm{D}$ problem and use an eighth-order scheme, the number of representative wavenumbers is $N_{k_{i}}^{2}=50^{2}=2500$.

Second, we reduce the number of times in solving the inverse problem (equation 16). In an isotropic heterogeneous medium, the locations with the same velocity have the same coefficients. Thus, it is only necessary to solve for the coefficients in equation 16 for $N_{v}$ times, where $N_{v}$ is the number of distinct velocities. The number of distinct velocities can be further reduced by discretizing the velocities based on a specified velocity increment that can be related to uncertainties in the velocity model. If we intend to control the inaccuracy under the uncertainty level in a velocity model, it only requires computing ETE coefficients on velocities with an a)

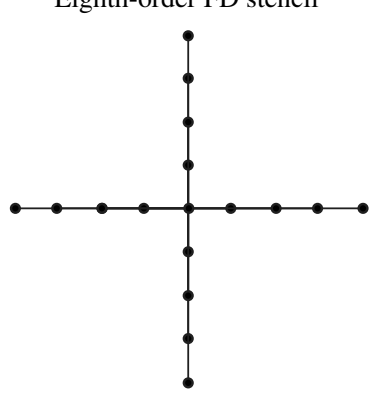

b) Eighth-order EWE stencil



Figure 1. Eighth-order (a) FD and (b) ETE stencil in 2D. The 2D ETE stencil has a cross-hair shape with off-axial points. interval twice the uncertainty. For instance, if the uncertainty is $2 \mathrm{~m} / \mathrm{s}$ in a velocity model that has a minimum velocity of $1.5 \mathrm{~km} / \mathrm{s}$ and a maximum velocity of $5.5 \mathrm{~km} / \mathrm{s}$, it only requires computing ETE coefficients at every $4 \mathrm{~m} / \mathrm{s}$. In such a case, $N_{v}$ is reduced to $10^{3}$ while the inaccuracy in velocity is $<0.2 \%$.

By choosing representative wavenumbers and computing ETE coefficients at distinct velocities, the total computational cost in
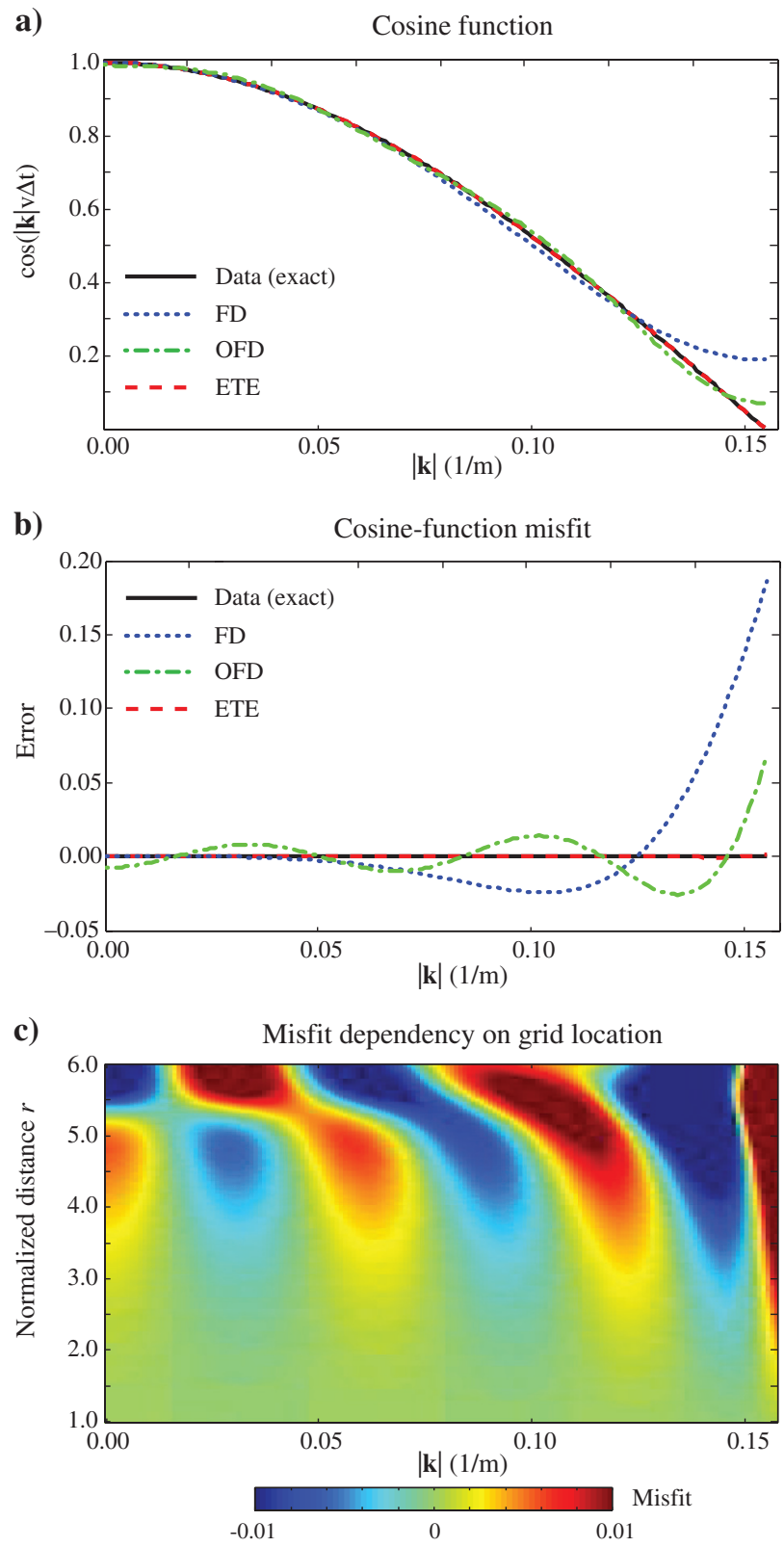

Figure 2. (a) Approximations of the cosine function (black solid line) in equation 14 using base functions corresponding to FD (blue dotted line), OFD (green dashed-dotted line), and ETE (red dashed line) methods. (b) The corresponding misfits of the three approximations (FD, blue dotted line; OFD, green dashed-dotted line; ETE, red dashed line). The parameters used in the simulations are $v=2 \mathrm{~km} / \mathrm{s}, \Delta t=5 \mathrm{~ms}, h=20 \mathrm{~m}, N_{a}=8$, and $N_{o}=2$. (c) Misfit computed from stencils with different off-axis grids, parameterized by the distance between off-axis grids and the stencil center $r$. 
solving the ETE coefficients is reduced to $O\left(N_{s}^{2} \cdot N_{k_{i}}^{n} \times N_{v}\right)$. Because the ETE coefficients only need to be computed once for a given velocity model, this cost is usually small compared to the propagation cost $O\left(N_{s} \cdot N_{x} \times N_{t}\right)$, where $N_{t}$ is the number of time steps. In isotropic media, the number of ETE coefficients is $N_{s} \cdot N_{v}$, which does not require significant memory.

\section{Wavenumber domain weighting}

The least-squares approach of equation 15 treats all the norms of wavenumbers equally. However, higher waveform accuracy may be achieved by introducing relative weights to different wavenumbers in the least-squares problem. For a single velocity, the dispersion relation associates wavenumbers with the source spectrum. Benefiting from the velocity discretization, we are able to formulate the weighting functions as

$$
\operatorname{wgt}(|\mathbf{k}|, v)=\frac{2 \pi A(f)^{2}}{v} \times \frac{1}{|\mathbf{k}|^{N-1}},
$$

where $A(f)$ is the source amplitude spectrum at frequency $f$. The reciprocal wavenumber term in equation 19 is introduced to decrease the weight of overrepresented large norms of wavenumbers. The weighting function in equation 19 decreases wavenumber with little energy and, as a result, helps to improve waveform accuracy.

\section{Cost of the wave propagation}

In ETE, the computational cost for one time step is approximately proportional to the number of stencil points. With minimal distance points, the ETE stencil has $N_{s}=n(L+2 n-2)+1$ for $2 \mathrm{D}$ and $3 \mathrm{D}$ problems. The extra cost of ETE is $\sim \frac{2(n-1)}{L}$ that of FD (e.g., $\sim 25 \%$ for the eighth-order $2 \mathrm{D}$ problem).

Although the ETE method is slightly more expensive than the FD method for each time step, its overall computational cost is less if compared with an FD method with same accuracy. In the second-order temporal FD method, a time step much smaller than the stability limit is often used to preserve waveform accuracy after iterating large numbers of time steps, whereas the ETE method tolerates time steps just below the stability limitation due to its high temporal accuracy.

\section{NUMERICAL EXAMPLES}

\section{Performance of the ETE method}

We first compare the ETE method with the conventional FD method in a 2D homogeneous medium with velocity of $2 \mathrm{~km} / \mathrm{s}$. We discretize the spatial grids in the $x$ - and $z$-directions at 20 and $25 \mathrm{~m}$ intervals, respectively. In a homogeneous medium, analytical waveforms at an arbitrary location can be obtained by shifting the source time function in time. We initialize wavefields at the first two time steps with analytical time shifts of a Ricker wavelet with a peak frequency of $10 \mathrm{~Hz}(\sim 35 \mathrm{~Hz}$ max frequency) and propagate wave. An eighth-order scheme is applied in FD and ETE methods to suppress spatial dispersion. In the ETE method, we select $\Delta t=6 \mathrm{~ms}$ and fit the target cosine (Figure 4a) by ETE stencil (Figure 1b) and ETE coefficients. In estimating the

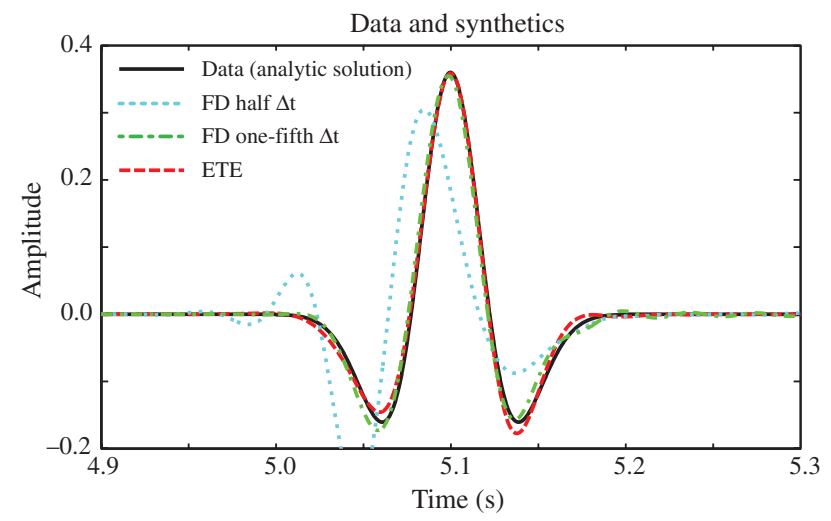

Figure 3. A comparison of synthetic waveforms in a homogeneous medium computed using FD and ETE. The cyan dotted line and the green dashed-dotted line represent FD synthetics calculated with a time step $\Delta t=3 \mathrm{~ms}$ and $\Delta t=1.2 \mathrm{~ms}$, respectively, and the red dashed line shows the ETE synthetic seismogram computed with a time step, $\Delta t=6 \mathrm{~ms}$. The black solid line indicates the analytic solution of the wave equation. The receiver offset is $10 \mathrm{~km}$, and the velocity of the medium is $2 \mathrm{~km} / \mathrm{s}$. a)


b)

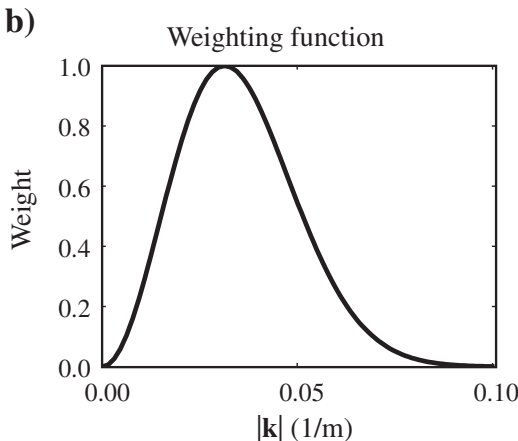

d)

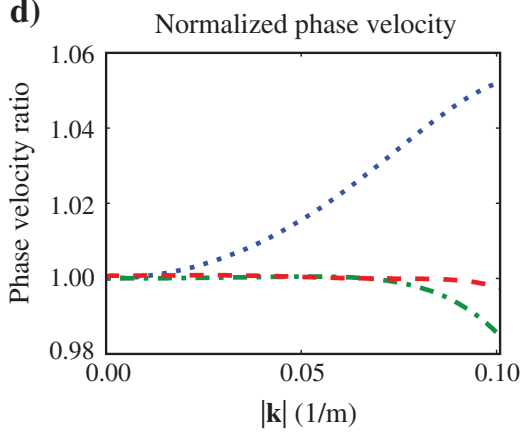

Figure 4. (a) Two target cosine functions corresponding to $\Delta t=6 \mathrm{~ms}$ and $\Delta t=1.2 \mathrm{~ms}$ are shown, respectively, in the red solid line and the green dashed-dotted line for comparison. (b) The wavenumber-domain weighting function computed from a Ricker source wavelet with a peak frequency of $10 \mathrm{~Hz}$. (c) Misfits of three implements of the target cosine functions. The blue dotted line and the green dashed-dotted line represent FD implements with a time step $\Delta t=6 \mathrm{~ms}$ and $\Delta t=1.2 \mathrm{~ms}$ respectively. The red dashed line shows the ETE misfits computed with a time step $\Delta t=6 \mathrm{~ms}$. (d) Relative phase velocity of the three implements. Line colors are similar to those in (c). 
ETE coefficients, we use $N_{k_{r}}=N_{k_{z}}=41$ representative wavenumbers with weights (Figure $4 \mathrm{~b}$ ) that corresponding to the source spectrum. The corresponding cosine function misfit (Figure $4 \mathrm{c}$ ) and phase velocity misfit (Figure 4d) are satisfactory. To demonstrate the temporal accuracy of the ETE method and the FD method, we choose to compare the modeled waveforms with the analytical waveform at a receiver with $10 \mathrm{~km}$ offset. We observe that the ETE waveform at this very far offset is similar to the reference waveform (Figure 3). On the other hand, the large misfits (Figure 4c and 4d) of FD with $\Delta t=6 \mathrm{~ms}$ cause unstable wave propagation. Even with $\Delta t=3 \mathrm{~ms}$, the FD waveform suffers from significant temporal inaccuracy (Figure 3). With $\Delta t=1.2 \mathrm{~ms}$, the FD method becomes fairly accurate in waveform (Figure 3), cosine function misfit (Figure 4c) and normalized phase velocity (Figure 4c). In this homogeneous case, the ETE method can achieve accuracy similar to the FD method with four times coarser time sampling. Counting the $\sim 25 \%$ extra cost in each time step and a negligible overhead, the ETE method saves the computational cost by $75 \%$ compared to conventional FD method.

To validate the local constant velocity assumption, we test the ETE performance in a two-layer velocity model with large velocity contrasts. We observe nonoscillatory reflection and refraction wavefronts from the boundary (Figure 5). It suggests that the ETE method is able to handle sharp velocity boundary. See the "Discussion" section for more detail.

To test the accuracy of the ETE method in a complex velocity model, we choose the Marmousi velocity model (Figure 6a). The Marmousi model is resampled to spatial grids with $20 \mathrm{~m}$ intervals in the $x$ - and $z$-directions. In ETE modeling, we discretize the true velocity model with a $4 \mathrm{~m} / \mathrm{s}$ interval from 1.5 to $5.5 \mathrm{~km} / \mathrm{s}$

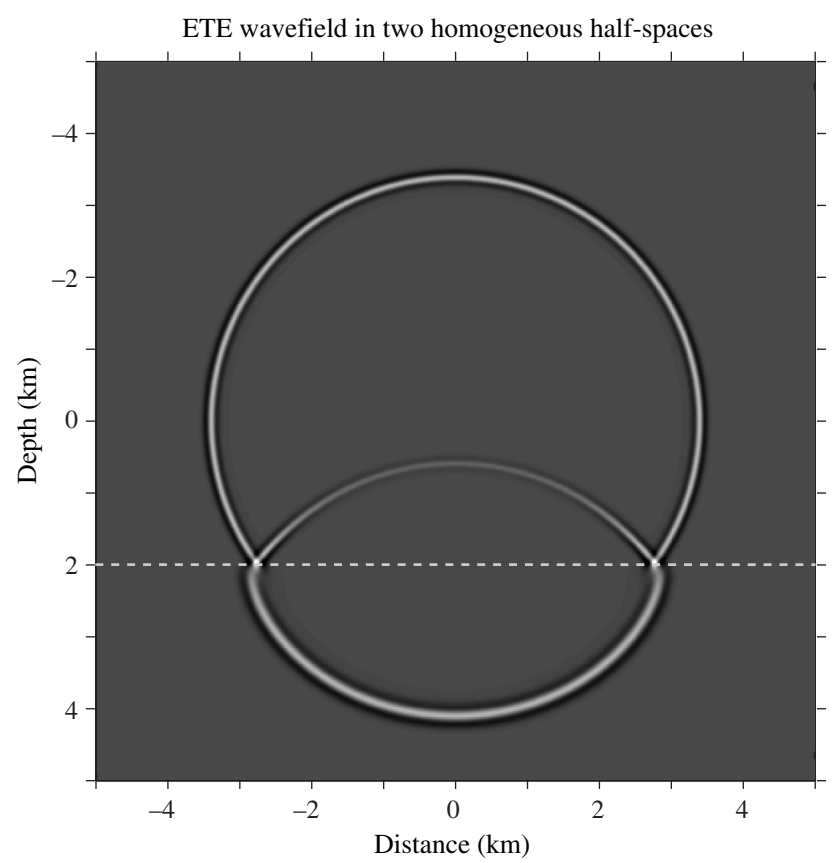

Figure 5. A snapshot of the ETE wavefield in a medium consisting of two uniform half-spaces. The wave speeds of the upper and lower half-space are 2.0 and $3.0 \mathrm{~km} / \mathrm{s}$, respectively. The time step and spatial grid sizes are $4 \mathrm{~ms}$ and $20 \mathrm{~m}$, respectively. The source is located inside the upper half-space at $(0,0)$ and is detonated at time 0 . and use 51 representative wavenumbers in each dimension. We insert a Ricker source (red star in Figure 6a) with a peak frequency of $10 \mathrm{~Hz}$ (with a maximum frequency of $\sim 35 \mathrm{~Hz}$ ) and select an eighthorder ETE scheme to suppress spatial dispersion. We implement an absorbing boundary condition (Clayton and Engquist, 1977) to suppress the reflections from the boundaries. We use $\Delta t=2 \mathrm{~ms}$, slightly smaller than the stability limit of $\sim 2.5 \mathrm{~ms}$, and propagate the ETE wave (Figure 6b). Note that the ETE wavefield is free of visible boundary reflections, suggesting its compatibility to an absorbing boundary condition. The target cosine function (Figure 7a) and ETE wavenumber domain weights (Figure 7b) are functions of velocity and the wavenumber norm. The cosine function misfit of the FD method (Figure 7c) is significantly larger than that of the ETE method (Figure 7d). The normalized FD phase velocity (Figure 7e) deviates much more than that of ETE (Figure 7f). The ETE

a)

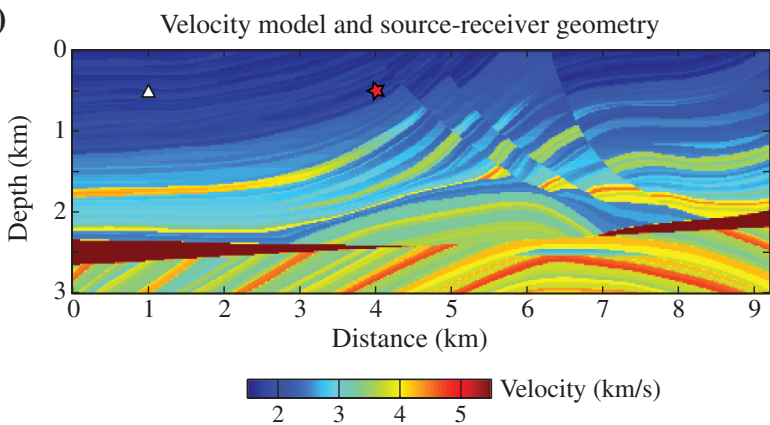

b)

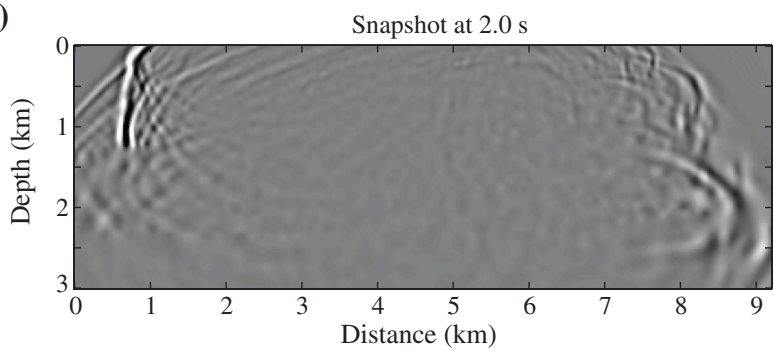

c)



Figure 6. (a) The Marmousi velocity model is plotted with an embedded source (red star) and receiver (white triangle), which indicates the location of the synthetic recordings shown in (c). (b) A snapshot of the ETE wavefield at $2.0 \mathrm{~s}$ after the detonation of the source. (c) The ETE (red dashed line) and FD (dashed-dotted line) synthetic seismograms are shown together with the anticipated true recording (data or reference waveform, black solid line). Both synthetics are computed with a time step of $2 \mathrm{~ms}$, and the data, or the reference waveform is computed using FD with a time step of $0.2 \mathrm{~ms}$. The differences between the synthetics and data are shown by the dotted lines: blue for FD and yellow for ETE. Note that the ETE seismogram matches the data much better than the FD synthetic does. 
waveform is compared with FD waveforms at the receiver (Figure $6 a)$. We choose to use the waveform computed by the FD method with a fine time step $\Delta t=0.2 \mathrm{~ms}$ as the reference waveform. With the same $\Delta t=2 \mathrm{~ms}$, almost no waveform difference is observed between the ETE waveform and the reference waveform, whereas noticeable waveform dispersion is observed on the FD waveform. In our implementation, the total computation time of the ETE simulation is $283 \mathrm{~s}$ on a quad-core desktop, including $58 \mathrm{~s}$ in finding the ETE coefficients and $225 \mathrm{~s}$ in wave propagation. On the other hand, the total computation time of the FD simulation is $174 \mathrm{~s}$ and that of the reference waveform simulation is $1742 \mathrm{~s}$. In this case, the memory used in storing the ETE coefficients is $\sim 84 \mathrm{~KB}$.
In the ETE method, the location dependent velocity $v(\mathbf{x})$ is assumed constant only in deriving the extrapolation error (equation 14), but it is not directly involved in the cosine function fitting approximation. The localized expansion in the cosine function fitting in obtaining the coefficients is similar to the Taylor series expansion of the FD method. Considering the Von Neumann stability limit, the localized constant velocity assumption is reasonable. As a result, we do not observe any oscillation at sharp velocity boundaries in our synthetic wavefield (Figure 5) and RTM images (Figure 8b).

The ETE method can potentially be extended to transversely isotropic media. Using the direction dependent velocity expression in Alkhalifah (1998), we expect that the ETE coefficients can be

\section{RTM image of the BP 2004 model using ETE}

We test the performance of the ETE method in RTM using the BP 2004 model (Billette and Brandsberg-Dahl, 2005). The BP data set is a high-quality synthetic data set generated using the FD method with shot and receiver spacing of 50 and $12.5 \mathrm{~m}$, respectively. In the migration, we use grid spacing of $12.5 \mathrm{~m}$ in the $x$ - and $z$ directions and set the maximum frequency at $35 \mathrm{~Hz}$. We choose an eighth-order ETE scheme to suppress the spatial dispersion and a time step of $1.3 \mathrm{~ms}$, slightly below the stability limit of $\sim 1.8 \mathrm{~ms}$. We use the true velocity model (Figure $8 \mathrm{a}$ ) to produce the section in Figure $8 \mathrm{~b}$. The top and base salt boundaries are well imaged, suggesting that the ETE method is able to handle sharp velocity boundaries, and the deep salt legs at $\sim 10 \mathrm{~km}$ in depth are imaged reasonably well, suggesting the high temporal accuracy of the ETE method.

\section{DISCUSSION}

In waveform modeling, reducing the number of points per wavelength is always computationally appealing because it reduces the size of a given problem. Unfortunately, limited sampling per wavelength may cause spatial dispersion for the same frequency content. Previous studies have shown that the spatial dispersion can be suppressed by using a higher order FD scheme (Dablain, 1986) or a full-size stencil in spectral implementation (Fomel et al., 2013). Because of the minimal number of grid points in a stencil, we use stencils with extra axial points to handle the spatial dispersion problem, similar to the FD scheme.

Violation of the constant-velocity assumption in deriving the Fourier integral may reduce the accuracy of wave extrapolation, mostly depending on the time sampling interval. Zhang and Zhang (2009) find that wave oscillation might occur at sharp velocity boundaries using the one-step extrapolation with a large time interval.
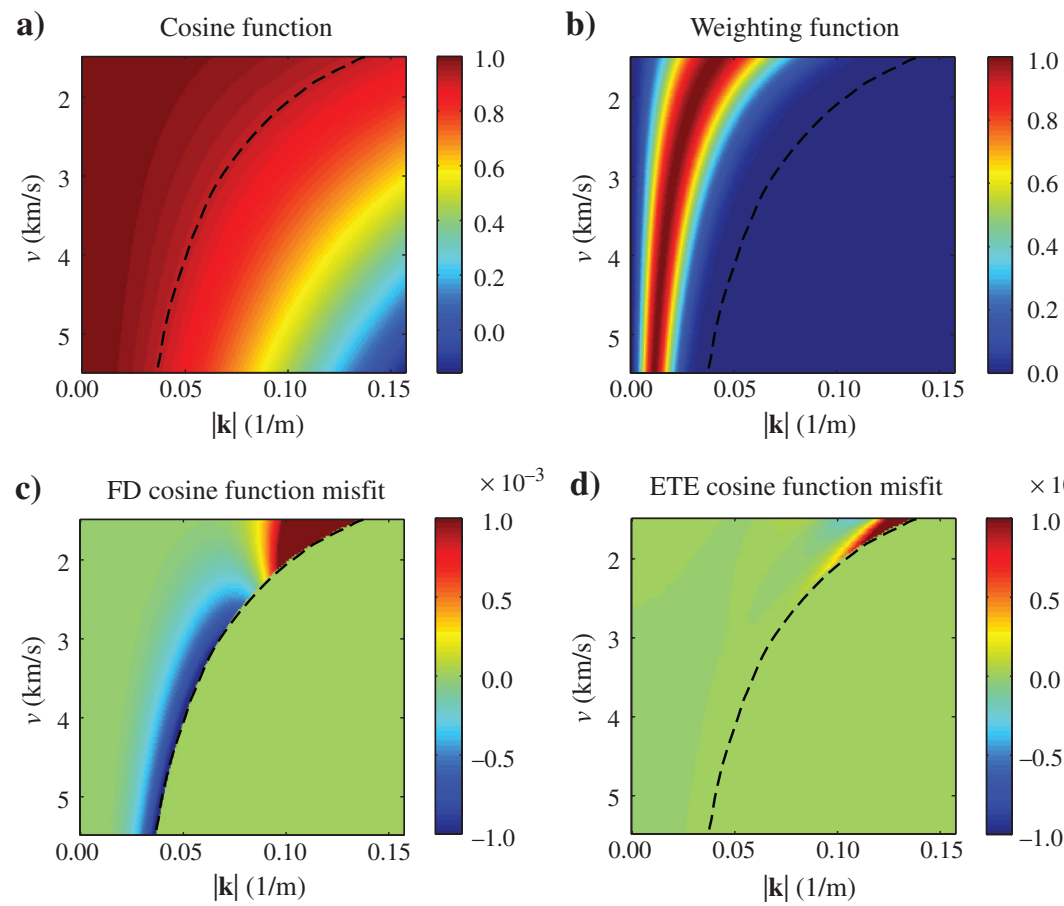

e) Normalized FD phase-velocity
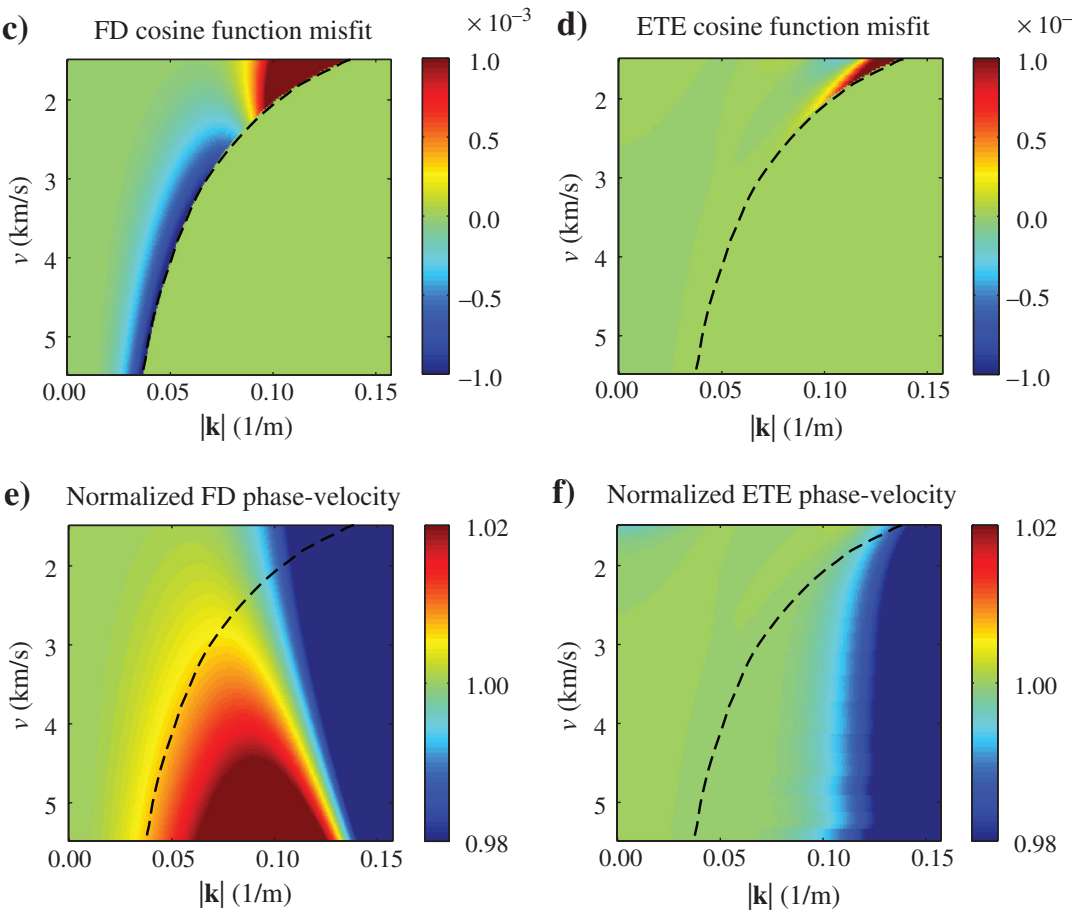

f) Normalized ETE phase-velocity

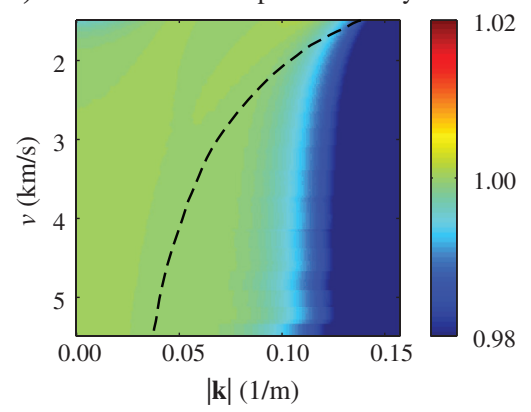

Figure 7. The target cosine function $(\cos (|\mathbf{k}| v \Delta t))$ and the weighting function of the Marmousi velocity model are plotted in color contour as a function of wavenumber and velocity in (a) and (b), respectively. The black dashed line marks the boundary between regions with zero (bottom-right) and nonzero (upper-left) weights. Panels (c, d) show the misfit of the target cosine function computed using the FD and ETE method, respectively. In both methods, the time step is $2 \mathrm{~ms}$. The misfits within the zero-weighting region are set to zero. Note that the ETE misfits are much smaller than the FD misfits in the effective region. Panels (e, f) show the relative phase velocity with respect to the true one in color contour in the $k, v$ domain computed using the FD and ETE methods, respectively. Note that the ETE method pushes the large phase velocity deviations to the zero-weighting region. 
a)

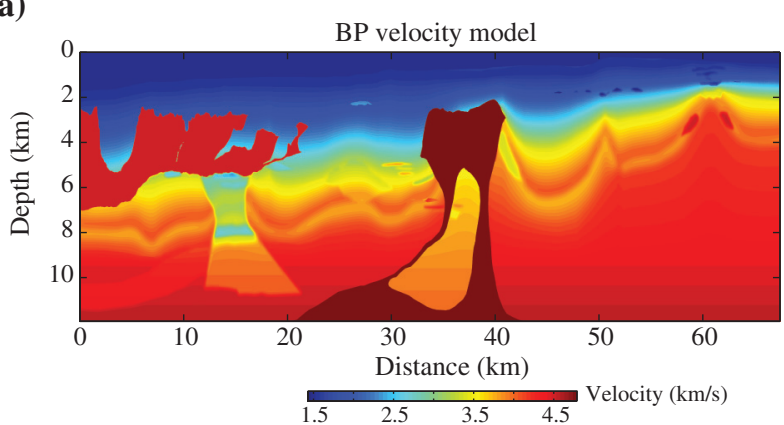

b)

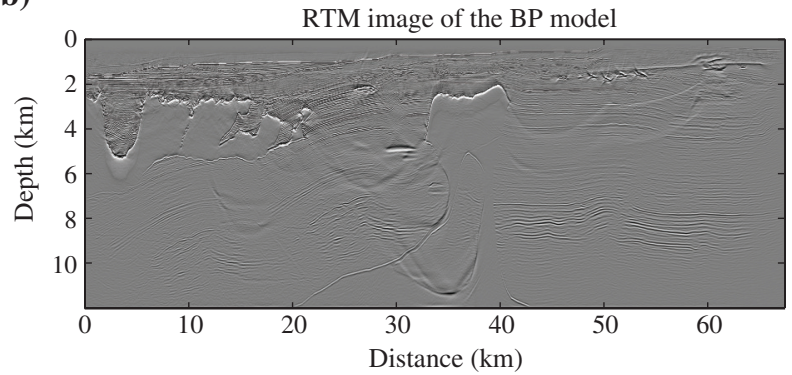

Figure 8. The BP 2004 velocity model (a) is shown together with the RTM image (b) computed with the ETE forward modeling scheme.

solved similarly with a substitution of velocity and wavenumbers. However, a dense stencil may be required to represent the skewed wavenumber-dependent behavior caused by a tilted symmetry axis. The optimization and performance of the ETE method in tilted transversely isotropic media is open to further research.

\section{CONCLUSIONS}

We present an ETE method to model wave propagation in acoustic media. The ETE method effectively provides accurate time evolution using optimum stencil and least-squares coefficients. The compact shape of the stencil ensures that high temporal accuracy is achieved with minimal cost. Under the Von Neumann stability condition, the ETE method remains stable and accurate through time. We suggest using the ETE method instead of the FD method for better accuracy and efficiency in isotropic acoustic waveform modeling in RTM and FWI.

\section{ACKNOWLEDGMENTS}

We thank A. Levander and W. Symes from Rice University; X. Song from UT Austin; C. Zeng from BGP International for helpful discussion; and four anonymous reviewers for their constructive comments and suggestions, which have significantly improved the manuscript. We also thank BGP for permission to publish this work. This research is supported by BGP and NSF grant EAR0748455 .

\section{REFERENCES}

Alkhalifah, T., 1998, Acoustic approximations for processing in transversely isotropic media: Geophysics, 63, 623-631, doi: 10.1190/1.1444361.

Alkhalifah, T., 2013, Residual extrapolation operators for efficient wavefield construction: Geophysical Journal International, 193, 1027-1034, doi: 10 .1093/gji/ggt040.

Baysal, E., D. D. Kosloff, and J. W. C. Sherwood, 1983, Reverse time migration: Geophysics, 48, 1514-1524, doi: 10.1190/1.1441434.

Billette, F. J., and S. Brandsberg-Dahl, 2005, The 2004 BP velocity benchmark: 67th Annual International Conference and Exhibition, EAGE Extended Abstracts, B035.

Carcione, J. M., G. C. Herman, and A. P. E. ten Kroode, 2002, Seismic modeling: Geophysics, 67, 1304-1325, doi: 10.1190/1.1500393.

Clayton, R., and B. Engquist, 1977, Absorbing boundary conditions for acoustic and elastic wave equations: Bulletin of the Seismological Society of America, 67, 1529-1540.

Dablain, M. A., 1986, The application of high-order differencing to the scalar wave equation: Geophysics, 51, 54-66, doi: 10.1190/1.1442040.

Dai, N., W. Wu, W. Zhang, and X. Wu, 2012, TTI RTM using variable grid in depth: Presented at IPTC 2012: International Petroleum Technology Conference.

Etgen, J., 1986, High-order finite-difference reverse time migration with the 2-way non-reflecting wave equation: Stanford Exploration Project, report SEP-48, 133-146.

Etgen, J., 1989, Accurate wave equation modeling: Stanford Exploration Project, report SEP-60, 131-148.

Fomel, S., L. Ying, and X. Song, 2013, Seismic wave extrapolation using lowrank symbol approximation: Geophysical Prospecting, 61, 526-536, doi: 10.1111/j.1365-2478.2012.01064.x.

McMechan, G. A., 1983, Migration by extrapolation of time-dependent boundary values: Geophysical Prospecting, 31, 413-420, doi: 10.1111/ j.1365-2478.1983.tb01060.x.

Pestana, R. C., and P. Stoffa, 2010, Time evolution of the wave equation using rapid expansion method: Geophysics, 75, no. 4, T121-T131, doi: 10.1190/1.3449091.

Song, X., and S. Fomel, 2011, Fourier finite-difference wave propagation: Geophysics, 76, no. 5, T123-T129, doi: 10.1190/geo2010-0287.1.

Song, X., S. Fomel, and L. Ying, 2013, Lowrank finite-differences and lowrank Fourier finite-differences for seismic wave extrapolation in the acoustic approximation: Geophysical Journal International, 193, 960969, doi: $10.1093 /$ gji/ggt017.

Soubaras, R., and Y. Zhang, 2008, Two-step explicit marching method for reverse time migration: 78th Annual International Meeting, SEG, Expanded Abstracts, 2272-2276.

Tal-Ezer, H., D. Kosloff, and Z. Koren, 1987, An accurate scheme for seismic forward modeling: Geophysical Prospecting, 35, 479-490, doi: 10 $.1111 / \mathrm{j} .1365-2478.1987 . \mathrm{tb} 00830 . \mathrm{x}$.

Tarantola, A., 1984, Inversion of seismic reflection data in the acoustic approximation: Geophysics, 49, 1259-1266, doi: 10.1190/1.1441754.

Tessmer, E., 2011, Using the rapid expansion method for accurate timestepping in modeling and reverse-time migration: Geophysics, 76, no. 4, S177-S185, doi: 10.1190/1.3587217.

Whitmore, N. D., 1983, Iterative depth migration by backward time propagation: 53rd Annual International Meeting, SEG, Expanded Abstracts, 382-385.

Zhang, Y., and G. Zhang, 2009, One-step extrapolation method for reverse time migration: Geophysics, 74, no. 4, A29-A33, doi: 10.1190/1 3123476 . 